\title{
Novel Structured Reporting Systems for Theranostic Radiotracers
}

\author{
Rudolf A. Werner ${ }^{1-3}$, Ralph A. Bundschuh ${ }^{4}$, Lena Bundschuh ${ }^{4}$, Stefano Fanti ${ }^{5}$, Mehrbod S. Javadi ${ }^{1}$, Takahiro Higuchi ${ }^{2,6}$, \\ Alexander Weich ${ }^{3,7}$, Kenneth J. Pienta ${ }^{8}$, Andreas K. Buck ${ }^{2,3}$, Martin G. Pomper ${ }^{1,8}$, Michael A. Gorin ${ }^{1,8}$, \\ Ken Herrmann ${ }^{9,10}$, Constantin Lapa*2, and Steven P. Rowe*1,8 \\ ${ }^{1}$ Division of Nuclear Medicine and Molecular Imaging, Russell H. Morgan Department of Radiology and Radiological Science, Johns \\ Hopkins University School of Medicine, Baltimore, Maryland; ${ }^{2}$ Department of Nuclear Medicine/Comprehensive Heart Failure \\ Center, University Hospital Würzburg, Würzburg, Germany; ${ }^{3}$ European Neuroendocrine Tumor Society Center of Excellence, NET \\ Zentrum, University Hospital Würzburg, Würzburg, Germany; ${ }^{4}$ Department of Nuclear Medicine, University Medical Center Bonn, \\ Bonn, Germany; ${ }^{5}$ Nuclear Medicine Unit, University of Bologna, S. Orsola Hospital Bologna, Bologna, Italy; ${ }^{6}$ Department of Bio \\ Medical Imaging, National Cardiovascular and Cerebral Research Center, Suita, Japan; ${ }^{7}$ Gastroenterology, Department of Internal \\ Medicine II, University Hospital Würzburg, Würzburg, Germany; ${ }^{8}$ James Buchanan Brady Urological Institute and Department of \\ Urology, Johns Hopkins University School of Medicine, Baltimore, Maryland; ${ }^{9}$ Department of Nuclear Medicine, University Hospital \\ Essen, Essen, Germany; and ${ }^{10}$ Department of Molecular and Medical Pharmacology, David Geffen School of Medicine at UCLA, Los \\ Angeles, California
}

\begin{abstract}
Standardized reporting is more and more routinely implemented in clinical practice, and such structured reports have a major impact on a large variety of medical fields, such as laboratory medicine, pathology, and, recently, radiology. Notably, the field of nuclear medicine is constantly evolving as novel radiotracers for numerous clinical applications are developed. Thus, framework systems for standardized reporting in this field may increase clinical acceptance of new radiotracers, allow for inter- and intracenter comparisons for quality assurance, and be used in global multicenter studies to ensure comparable results and enable efficient data abstraction. In the last couple of years, several standardized framework systems for PET radiotracers with potential theranostic applications have been proposed. These include systems for prostate-specific membrane antigen-targeted PET agents to diagnose and treat prostate cancer, and systems for somatostatin receptor-targeted PET agents to diagnose and treat neuroendocrine neoplasia. In the present review, the framework systems for these 2 types of cancer will be briefly introduced, followed by an overview of their advantages and limitations. In addition, potential applications will be defined, approaches to validate such concepts will be proposed, and future perspectives will be discussed.
\end{abstract}

Key Words: prostate cancer; neuroendocrine neoplasia; ${ }^{68} \mathrm{Ga}-$ DOTATATE; ${ }^{8} \mathrm{Ga}$-DOTATOC; ${ }^{88} \mathrm{Ga}$-DOTANOC; somatostatin receptor

J Nucl Med 2019; 60:577-584

DOI: 10.2967/jnumed.118.223537

Received Nov. 14, 2018; revision accepted Dec. 29, 2019.

For correspondence or reprints contact: Steven P. Rowe, Division of Nuclear Medicine and Molecular Imaging, Russell H. Morgan Department of Radiology and Radiological Science, Johns Hopkins University School of Medicine, 601 N. Caroline St., Baltimore, MD 21287.

E-mail: srowe8@jhmi.edu

${ }^{*}$ Contributed equally to this work.

Published online Feb. 22, 2019.

COPYRIGHT (c) 2019 by the Society of Nuclear Medicine and Molecular Imaging.
I general, standardized reporting has recently become implemented more routinely in clinical practice. Such structured reports, which, in some contexts, were introduced as early as the 1970s, have had a major impact on a large variety of medical fields, such as laboratory medicine (1), pathology (2), and, recently, radiology $(3,4)$. Notably, the field of nuclear medicine is constantly evolving as novel radiotracers for numerous clinical applications are developed and administered in the clinical setting (5-8). Thus, framework systems for standardization of reporting in this field would be particularly necessary. Nuclear medicine reporting frameworks may increase clinical acceptance of novel radiotracers that are being transitioned into routine clinical practice, open avenues for inter- and intrareader and inter- and intracenter comparisons for quality assurance purposes, and be used in national or global multicentric studies to more efficiently enable comparison of datasets from different centers.

In the last 2 decades, somatostatin receptor (SSTR) agonists such as ${ }^{68} \mathrm{Ga}$-DOTATOC, ${ }^{68} \mathrm{Ga}$-DOTATATE, and ${ }^{68} \mathrm{Ga}$-DOTANOC and subsequent therapies with either ${ }^{177} \mathrm{Lu}$ - or ${ }^{90}$ Y-labeled analogs have become a standard of care for patients with SSTR-expressing tumors throughout the world (9-12). In a similar vein to SSTRtargeted PET for neuroendocrine neoplasia (NEN), the use of radiotracers for prostate-specific membrane antigen (PSMA), labeled with either ${ }^{68} \mathrm{Ga}$ or ${ }^{18} \mathrm{~F}$, is rapidly becoming a new standard of care for prostate cancer (PCa) imaging throughout much of the world, in particular because the theranostic concept can potentially be applied in PCa as well (13-16).

To aid interpretation of ${ }^{18} \mathrm{~F}-\mathrm{FDG}$ PET findings in determining oncologic response to therapy, there have been several proposed systems, such as the Deauville/Lugano criteria and PERCIST 1.0. These systems are specifically less focused on providing diagnostic information on baseline imaging studies $(17,18)$. However, in light of the evolving field of theranostics for NEN and $\mathrm{PCa}$, in which findings on baseline diagnostic imaging portend treatment success with radioligand therapies, the field is in need of baseline criteria for evaluating either PSMA- or SSTR-targeted PET scans. 
This clinical need has not gone unnoticed, and a great deal of recent progress has been made by the introduction of novel standardized framework systems for the evaluation of various receptorbased radiotracers. For PCa, these systems include the PSMA Reporting and Data System (RADS), version 1.0; the Prostate Cancer Molecular Imaging Standardization Evaluation (PROMISE); and a proposed standardized framework system for ${ }^{68} \mathrm{Ga}$-PMSA $\mathrm{PET} / \mathrm{CT}$ for the detection of recurrent lesions, which was written on behalf of the European Association of Nuclear Medicine (EANM) (19-21) and is referred to here as the EANM Consensus Paper (21). For NEN, the framework systems include SSTRRADS, version 1.0, which applies the concept of PSMA-RADS to NEN and considers SSTR-specific details (22). Notably, both RADS framework systems (PSMA- and SSTR-RADS) have been recently summarized under the umbrella term molecular imaging (MI) $R A D S$, as these systems can be applied reciprocally (i.e., imaging interpreters who are familiar with one RADS system should be able to learn the other) (23). Another NEN-specific system is the NETPET grade, which uses a dual-radiotracer approach (SSTR-targeted and ${ }^{18}$ F-FDG PET). The NETPET scoring system will also be reviewed here; however, this system has been developed as a prognostic biomarker to capture the findings of a dual-radiotracer assessment in a single parameter rather than as a method for evaluating a baseline SSTR-PET scan (24).

In the present review, these concepts will be briefly introduced, followed by an overview of their advantages and limitations. In addition, potential applications will be defined, approaches to validate such concepts will be proposed, and future perspectives will be discussed.

\section{PATIENT POPULATION}

Because the data presented here comprise a retrospective analysis of routinely acquired data, the local ethic committee waived the need for further approval. All patients gave written informed consent to the procedures and to scientific analysis of the obtained data.

\section{STANDARDIZED REPORTING SYSTEMS FOR PCA}

\section{EANM Consensus Paper}

On behalf of the EANM, Fanti et al. (21) proposed a standardized imaging interpretation system for ${ }^{68} \mathrm{Ga}$-PSMA that uses the following criteria: first, anomalous findings, defined as suggestive radiotracer uptake above physiologic background, are recorded. Second, all these sites are classified as "pathologic" for PCa, unless another explanation is apparent, that is, false-positive findings, such as PSMA-avid Paget disease (25). Third, the anatomic localization is considered (up to 5 lesions). Of note, Fanti et al. not only

\section{NOTEWORTHY}

- Several framework systems for both PSMA-targeted PET for PCa and SSTR-targeted PET for NEN have been introduced.

- For PSMA-targeted PET, these systems consist of a consensus paper written on behalf of the EANM, PSMA-RADS, and PROMISE.

- For SSTR-targeted PET, these systems consist of the NETPET grade and SSTR-RADS.

- Future directions should focus on validating such systems or defining a single universal framework system per radiotracer. proposed the system but also performed a concordance assessment among multiple readers. Notably, an interobserver agreement (Krippendorf $\alpha$ ) of 0.64 (i.e., moderate agreement) was achieved when readers had to evaluate whether the investigated target lesions were suggestive of a pathologic, uncertain, or nonpathologic entity. After conducting several Delphi rounds to reach consensus between the different study sites, the following amendments were introduced: first, a finding observed only on CT will be classified as "abnormal" but "uncertain"; second, in patients with residual prostatic tissue (no radical prostatectomy) and sources of artifact (e.g., brachytherapy seeds), the intensity of focal uptake is important in deciding whether the lesion should be classified as "pathologic"; and third, recurrence sites (other than the site of primary treatment) need increased attention and, again, in cases of intense focal uptake, can be considered "pathologic."

This framework system has also been further validated: in 49 subjects from 7 study sites, a moderate agreement among readers was achieved. Notably, several Delphi rounds were conducted and led to further improvement of the system, reduced the number of discordant cases, and achieved consensus among the readers. Thus, the EANM consensus paper aimed to identify possible disagreements among multiple experts and provide suggestions for appropriate reporting (21). However, a definition for the level of uptake, as defined in PROMISE, is not given. Further, a translation of the derived findings into an alphanumeric code, as suggested in PSMA-RADS, may facilitate memorizing this system (19-21). Nonetheless, as a major achievement of the EANM consensus paper, the authors provide a definition of the findings that can be seen as uncertain, which, in turn, may lay the groundwork for fusing this system with the 5-point scale provided in PSMA-RADS and the level of uptake as defined in PROMISE $(20,21)$.

\section{MI-RADS}

A recent effort has summarized 2 RADS framework systems proposed to date for MI (i.e., PSMA- and SSTR-targeted PET/CT interpretation, namely PSMA- and SSTR-RADS) under a single umbrella term: MI-RADS (23). MI-RADS systems are exclusively based on imaging findings (site of disease and intensity of radiotracer uptake), and both refer to a 5-point Likert scale (from 1, no evidence of disease/definitively benign, to 5 , high certainty that $\mathrm{PCa}$ or NEN is present). This underlying identical structure allows both MI-RADS systems to be used reciprocally (23). Supplemental Table 1 provides a precise overview of MI-RADS (supplemental materials are available at http://jnm.snmjournals.org). In the following, we will first introduce PSMA-RADS for PCa.

\section{PSMA-RADS, Version 1.0}

Rowe et al. suggested a 5-point scale for the interpretation of PSMA-targeted PET/CT for PCa and also provided recommendations for appropriate next steps in the work-up of indeterminate findings (19). In brief, PSMA-RADS-1A describes a scan without abnormal radiotracer uptake (i.e., normal biodistribution of a PSMA imaging agent), whereas PSMA-RADS-1B findings are benign lesions that demonstrate radiotracer uptake and have been confirmed by histologic diagnosis or definitively characterized as benign on imaging (e.g., a PSMA-avid thyroid nodule that has been previously biopsied and found to be an adenoma). PSMA-RADS-2 describes sites with low-level uptake (no more than the blood pool level), which are almost certainly benign and would be atypical for PCa. PSMA-RADS-3 includes indeterminate lesions, and thus, these lesions may trigger further work-up. In PSMA-RADS-4 lesions, PCa 
is highly likely because of intense uptake in a site typical for PCa but without an abnormality on anatomic imaging. PSMA-RADS-5 lesions demonstrate intense PSMA uptake in a site typical for PCa, with corresponding evidence on conventional imaging, and $\mathrm{PCa}$ is almost certainly present (Supplemental Table 1) (19). As proposed in its successor, SSTR-RADS (22), an overall PSMA-RADS score assessment may be useful to provide the referring clinician with an overall scan impression in the summary statement or impression of the report. Further, up to 5 target lesions are selected and given individual PSMA-RADS scores. On the basis of this target lesion assessment, the overall PSMA-RADS score can be defined as the highest score of any of the individual target lesions. Figure 1 shows an overall PSMA-RADS score assessment.

Notably, the PSMA-RADS scoring system has recently been further validated. PSMA-RADS-3A (soft-tissue sites) and -3B (bone lesions) can refer to findings that have low levels of uptake and lack a correlative anatomic finding (19). In patients with indeterminate findings and available follow-up imaging, the majority $(75.0 \%)$ of PSMA-RADS-3A lesions demonstrated changes on subsequent imaging compatible with the presence of $\mathrm{PCa}$, whereas only a minority $(21.4 \%)$ of PSMA-RADS-3B indeterminate bone lesions showed changes on follow-up imaging suggestive of underlying PCa. These findings confirm the necessity for a category in the PSMA-RADS grading system for indeterminate lesions (26). In addition, the PSMA-RADS system has also been evaluated in a prospective interobserver agreement setting using $50{ }^{18} \mathrm{~F}-\mathrm{DCFPyL}$ PET/CT scans. Four readers ( 2 experienced and 2 inexperienced), masked to the clinical status of the patients, performed an evaluation of all scans independently and evaluated PSMA-RADS on a target lesion-based, an organ-based, and an overall PSMA-RADS score-based level. The interobserver agreement for PSMA-RADS scoring among identical target lesions was good (intraclass correlation coefficient, $\geq 0.60$ ). For lymph nodes and the overall scan impression (Fig. 2), an excellent interobserver agreement was derived (intraclass correlation coefficient, 0.79 and 0.84 , respectively). Given the high concordance rate in this study, even among readers with different levels of experience, PSMA-RADS may be able to be implemented in the collection of data for large prospective trials (27).
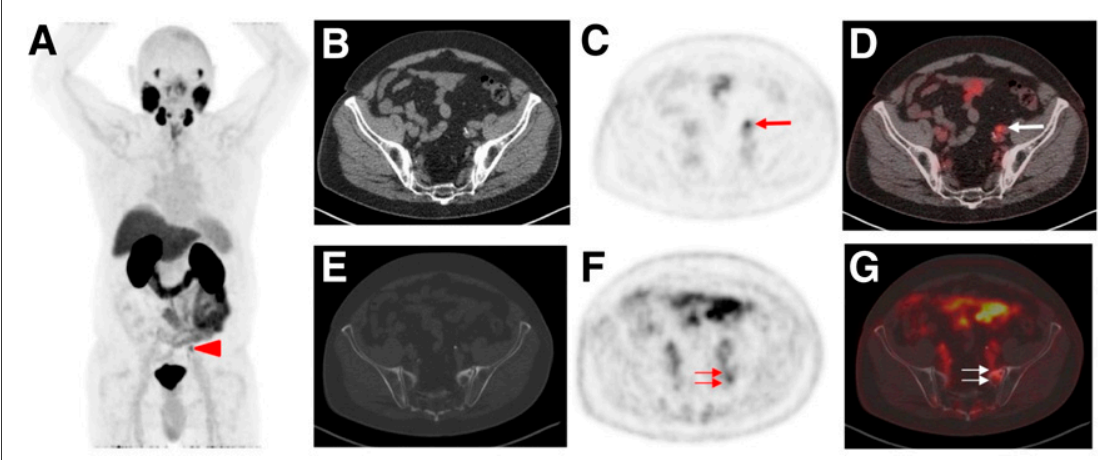

FIGURE 1. Example of overall assessment using PSMA-RADS, version 1.0 (19) in 76-y-old man with history of proven PCa who underwent ${ }^{18} \mathrm{~F}-\mathrm{DCFPyL} \mathrm{PET} / \mathrm{CT}$ for staging. (A) Whole-body maximum-intensity projection demonstrates suggestive radiotracer uptake (arrowhead). (B-D) Visualization of uptake in left external iliac lymph node (arrows) on axial CT (B), PET (C), and PET/CT (D). Experienced reader classified this uptake as PSMA-RADS-4 because on CT there is no corresponding pathologic finding. $(E-G)$ Because equivocal uptake in bone lesion would not be atypical on anatomic imaging for $\mathrm{PCa}$, finding in ala of left sacrum (arrows) on axial CT (E), PET (F), and PET/CT (G) was classified as PSMA-RADS-3B. Overall RADS score was 4 (Supplemental Table 1).

\section{PROMISE}

Eiber et al. proposed an MI-TNM classification ("miTNM," version 1.0) as a standardized framework system for PSMA-ligand PET/CT and PET/MRI (20). This scoring system determines the uptake on a 4-point scale in visual assessment ("miPSMA expression score," with different levels of uptake noted relative to the mal uptake in the blood pool, liver, and parotid glands). Of PET agents, such as ${ }^{18}$ F-PSMA 1007, the liver is replaced by the spleen $(20,28)$. Moreover, categorization of local tumor takes the xtent and organ confinement into account (miT0, no local tumor; 2 , organ-confined tumor with " $\mathrm{u}$ " [unifocality] or "m" [multilar extension] or "b" [tumor invading seminal vesicles]; miT4, tumor invading adjacent structures other than seminal vesicles). A strength of PROMISE is that it directly addresses local tumor staging. To evaluate the intraprostatic tumor extension, a sextant y provide detailed information for biopsies.

Meover, in PROMISE, the pelvic node metastases are assessed $\mathrm{miN1a} / \mathrm{b}$ : II (internal iliac), EI (external iliac), CI (common iliac), OB (obturator), PS (presacral), or OP (other pelvic [should be specified]). Finally, the extrapelvic nodes are included (miM1a: RP [retroperitoneal], SD [supradiaphragmatic], or OE [other extrapelvic]), as well as distant metastases (miM1b [referring to bone] or miM1c [referring to other sites of organ involvement]). Skeletal involvement is classified as being unifocal (Uni), oligometastatic igo, $\leq 3$ sites), disseminated (Diss), or diffuse bone marrow . The authors recommend documenting diagnostic cerof disease"). In addition, the final diagnosis should be reported as positive for $\mathrm{PCa}$, negative for $\mathrm{PCa}$, or, if further work-up using ther techniques is available, equivocal for PCa. Taken together, MISE includes information on location, disease distribution pattern, level of PSMA expression, and level of certainty that $\mathrm{PCa}$ is present. Figure 3 gives an example of primary staging using ${ }^{68} \mathrm{Ga}-\mathrm{PSMA}-11$ PET/high-resolution T2-weighted MRI in a 65-y-old man with histopathologically proven $\mathrm{PCa}$. PROMISE has been applied to this patient with a result of miT3aN1(OBL)M0 (with L referring to the left side of obturator lymph node involvement) (20).

\section{Potential Applications}

Similar to ${ }^{18} \mathrm{~F}-\mathrm{FDG}$, a considerable number of studies have reported pitfalls in reading PSMA-targeted PET scans. As a physiologic radiotracer uptake site, ganglia can be misinterpreted, in particular in terms of mistaking such structures for abdominal lymph nodes (e.g., celiac ganglia) (29-31). Benign pathologic states may also mimic $\mathrm{PCa}$, such as fibrous dysplasia, healing bone fractures, or granulomatous diseases (e.g., sarcoidosis) (25,32-34). Nonprostatic malignancies that may demonstrate uptake of PSMAtargeted radiotracers include, but are not 


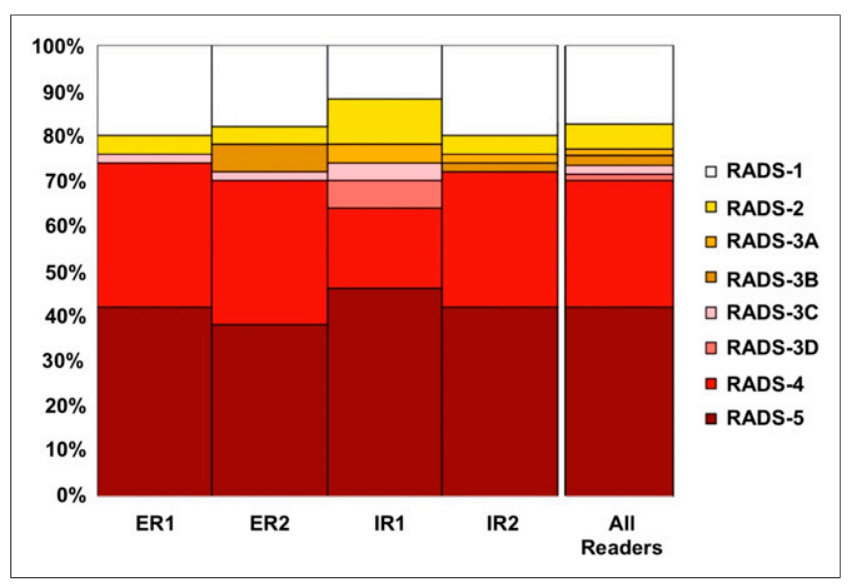

FIGURE 2. Overall PSMA RADS scoring for 4 masked readers (ER $=$ experienced reader; IR = inexperienced reader), all of whom evaluated $50{ }^{18} \mathrm{~F}-\mathrm{DCFPyL}$ PET/CT scans. Often, characterizing lesion as PSMARADS-1B involves previous conventional imaging or histologic diagnosis; as such, PSMA-RADS-1A and -1B were subsumed under PSMA-RADS1 in this masked interobserver agreement study. For overall scan impression, high interreader agreement rate, even among IRs, was noted.

limited to, pancreatic NEN, squamous cell carcinoma of the oropharynx, hepatocellular carcinoma, and renal cell carcinoma $(25,35-38)$. Although the detection rate and recognition of potentially confusing false-positive or false-negative findings on PSMA-targeted PET scans can almost certainly be increased by appropriate training, the way in which PSMA-RADS may live up to its full potential is in providing readers with a mechanism to formalize uncertainty about such findings and to recommend any (Reprinted from (27).)

further workup to clinicians. Intended to serve as a readily applicable system for any imaging expert, PSMA-RADS is simple and easy to memorize and use and, thus, may serve as a powerful tool to assist in diagnosis in a busy clinical PCa practice. However, as a drawback, PSMA-RADS uses terms such as typical or atypical for sites of disease, and follow-up versions of this system should clarify such phrases to increase the reader's confidence $(19,39)$. PSMA-RADS does not stipulate the inclusion of in-depth anatomic details, unlike the EANM consensus paper and PROMISE, which thoroughly evaluate PSMA-targeted PET/CT or PET/MRI findings and include many details on imaging interpretation $(20,21)$. This characteristic may pave the way for incorporating the latter systems into large clinical trials, in which distinct, numerous parameters should be obtained to enrich the dataset and allow for fine parsing of patient subsets for outcomes research. PROMISE also includes visual criteria using an internal organ reference of PSMA uptake-a consideration that, although requiring further confirmation, may persuade principal investigators to apply PROMISE in a research setting $(20,39)$. In addition, threshold sensitivity may also have an impact on the miPSMA expression score (39). PROMISE is the only system reviewed here that has been used in a preclinical setting to investigate the in vivo relationship between ${ }^{68} \mathrm{Ga}$-PSMA-11 PET/CT and PSMA expression in a murine model of PCa (40). A major advantage of the system proposed by the EANM is that it has been assessed in a Delphi approach of consensus, which serves as a reliable means for categorizing tumor entities $(21,41)$ and may be a significant advantage for training less experienced readers. Currently, all PCa framework systems lack treatment recommendations for endoradiotherapies. However, given the evolving field of ${ }^{68} \mathrm{Ga} /{ }^{18} \mathrm{~F} /{ }^{177} \mathrm{Lu}$-PSMA theranostics, future versions should consider providing instructions on when to initiate such treatments.

Table 1 summarizes limitations and advantages of framework systems for evaluating PSMA-targeted PET/CT scans for PCa patients.
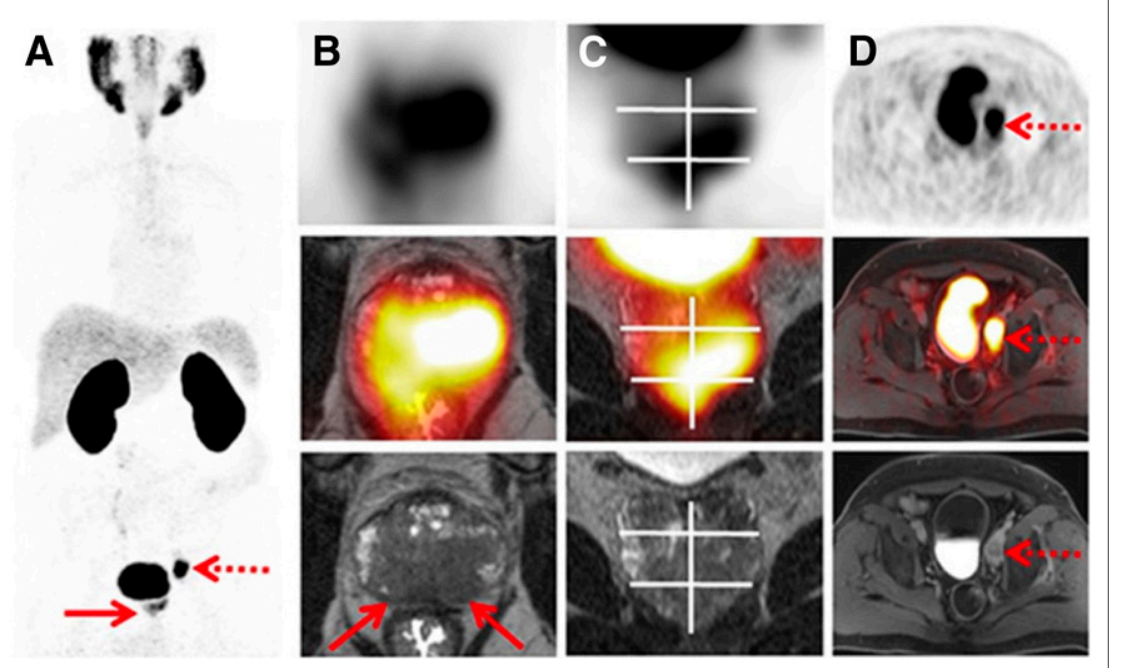

FIGURE 3. Example of PROMISE (20) for primary staging using ${ }^{68} \mathrm{Ga}-\mathrm{PSMA}-11 \mathrm{PET} / \mathrm{high}$ resolution T2-weighted MRI in 65-y-old man with histopathologically proven $\mathrm{PCa}$. (A) Maximum-intensity projection shows intermediate PSMA expression (equivalent to score 2) in prostate gland (solid arrow) and high PSMA expression (score 3) in regional pelvic lymph node (dotted arrow). (B and C) Axial (B) and coronal (C) PET/MRI, with PET at top, hybrid imaging in middle, and MRI at bottom, demonstrate bilateral T2-hypointense lesions that correspond to uptake on PSMA-ligand PET and exceed prostate margin, indicating extracapsular extension (T3a, arrows). (D) Axial imaging shows single lymph node metastasis in left obturator region (arrows). Final diagnosis according to PROMISE was miT3aN1(OBL)M0. Sextant segment boundaries are shown on coronal images in white. (Reprinted from (20).)

\section{STANDARDIZED REPORTING SYSTEMS FOR NEN}

\section{The NETPET Grade}

In contrast to the framework systems for $\mathrm{PCa}$, the NETPET grade intends not to evaluate an isolated baseline examination but to provide a dual SSTR $/{ }^{18}$ F-FDG grading scheme. This intention is mainly due to the assumption that the most ${ }^{18}$ F-FDG-avid lesion (relative to its uptake on SSTR-PET) may also reflect the most aggressive phenotype of the disease present. On a 5-point scale, the spectrum of results seen on both PET scans is as follows: P0 is negative on both PET studies; P1 is SSTR-positive but ${ }^{18}$ F-FDG-negative; P2 through P4 are positive on both PET studies, but their intensities relative to each other differ (i.e., these lesions represent an intermediate group on dual-radiotracer imaging); and P5 is SSTRnegative but ${ }^{18}$ F-FDG-positive $(24,42)$. Notably, analogous to the EANM consensus paper on PSMA-targeted PET, the NETPET 
TABLE 1

Head-to-Head Comparison of Standardized Framework Systems for PSMA PET/CT for Evaluating PCa

\begin{tabular}{|c|c|c|}
\hline Standardized framework system & Advantages & Limitations \\
\hline EANM consensus paper (21) & $\begin{array}{l}\text { Is a consensus strategy for validation: after } \\
\text { initial introduction, system has been } \\
\text { validated by different sites and further } \\
\text { improved in Delphi consensus setting } \\
\text { Provides detailed evaluation and criteria for } \\
\text { uncertain findings } \\
\text { Without having a scale, demonstrated } \\
\text { moderate consensus among experts } \\
\text { and, thus, emphasizes importance of } \\
\text { incorporating scalability in structured } \\
\text { reporting systems }\end{array}$ & $\begin{array}{l}\text { Lacks visual criteria scale, e.g., based on } \\
\text { internal organ uptake as reference } \\
\text { Does not include treatment recommendations } \\
\text { for PSMA-based endoradiotherapies }\end{array}$ \\
\hline PSMA-RADS, version 1.0 (19) & $\begin{array}{l}\text { Considers site of disease and intensity of } \\
\text { radiotracer uptake on 5-point scale, which } \\
\text { may be easy to memorize } \\
\text { Is part of MI-RADS; i.e., imaging interpreters } \\
\text { who are familiar with PSMA-RADS should be } \\
\text { able to learn SSTR-RADS (22,23) } \\
\text { May be useful to guide reader in interpreting } \\
\text { confusing false-positive or -negative } \\
\text { discoveries, e.g., potential nonprostatic } \\
\text { malignancies or benign findings mimicking } \\
\text { PCa (25) } \\
\text { Provides clinical recommendations based on } \\
\text { PSMA-RADS scoring (e.g., PSMA-RADS-3 } \\
\text { triggers further work-up) (39) } \\
\text { Has been further validated in long-term follow- } \\
\text { up study investigating indeterminate PSMA- } \\
\text { RADS-3A and -3B lesions (26) } \\
\text { Demonstrated high interobserver agreement, } \\
\text { even among inexperienced readers (27) }\end{array}$ & $\begin{array}{l}\text { Needs further definition of terms such as typical } \\
\text { or atypical for PCa in follow-up version (39) } \\
\text { Needs overall PSMA-RADS score definition, } \\
\text { similar to SSTR-RADS (22) } \\
\text { Lacks visual criteria scale, e.g., based on } \\
\text { internal organ uptake as reference } \\
\text { Does not include treatment recommendations } \\
\text { for PSMA-based endoradiotherapies } \\
\text { Has arbitrary initial definitions and needs further } \\
\text { validation, e.g., by comparison of PSMA- } \\
\text { RADS classification with histologic specimen } \\
\text { or outcome assessments }\end{array}$ \\
\hline PROMISE (20) & $\begin{array}{l}\text { Considers anatomic details, e.g., tumor } \\
\text { location on sextant basis } \\
\text { Provides visual criteria scale (miPSMA } \\
\text { expression score) based on reference organs } \\
\text { Initially invented with intention to be applicable } \\
\text { to both PET/CT and PET/MRI } \\
\text { Has variety of included details that may pave } \\
\text { way for incorporation in large clinical trials, in } \\
\text { which numerous parameters are needed to } \\
\text { enrich dataset } \\
\text { Was tested in vivo in PCa murine model and, } \\
\text { thus, could potentially be applied in other } \\
\text { preclinical settings (40) }\end{array}$ & $\begin{array}{l}\text { Needs further validation, e.g., interobserver } \\
\text { agreement studies and histopathologic } \\
\text { comparisons } \\
\text { Has a threshold sensitivity that may affect } \\
\text { miPSMA expression score (39) } \\
\text { Does not include treatment recommendations } \\
\text { for PSMA-based endoradiotherapies }\end{array}$ \\
\hline
\end{tabular}

grade has been validated in the same paper $(21,24)$. Kaplan-Meier curves presented for subjects grouped to P1, P2-P4, and P5 revealed a significant separation of overall survival for those 3 groups, indicating that the NETPET grade may serve as a predictor for outcome. However, the study cohort included different sites of primary tumor and different treatments (only parts of the cohort were treated with peptide receptor radionuclide therapy [PRRT]) (24).

\section{SSTR-RADS, Version 1.0}

Analogous to PSMA-RADS, a standardized framework system for interpreting SSTR-targeted PET/CT scans has recently been introduced and has been given the moniker SSTR-RADS (22). As part of MI-RADS (23), SSTR-RADS also uses a 5-point scale (1, benign, to 5, NEN almost certainly present) and is based exclusively on imaging findings (site of disease and intensity of radiotracer uptake); however, it refers to SSTR-targeted PET and NEN-specific details. SSTR-RADS-1 indicates findings that are known to be benign (confirmed by previous biopsy or with a pathognomonic appearance on conventional anatomic imaging) and includes SSTR-RADS-1A (normal biodistribution) and SSTR-RADS-1B (increased focal uptake but definitively benign by histology or imaging). SSTR-RADS-2 lesions are likely benign and describe soft-tissue sites or bone lesions atypical for metastatic NEN (e.g., strongly suspected to be degenerative, such as a Schmorl node). SSTR-RADS-3 includes indeterminate lesions and will often require further work-up. SSTR-RADS-4 (NEN highly likely) includes intense uptake in a site typical for NEN but lacks definitive findings on CT. SSTR-RADS-5 indicates a lesion in 
which NEN is almost certainly present, and the site of intense radiotracer uptake on SSTR-PET corresponds to an anatomic abnormality (Supplemental Table 1). Those SSTR-RADS classifications not only have recommendations for further work-up (biopsy or imaging) but also propose at which time-point to initiate PRRT with either ${ }^{177} \mathrm{Lu}$ - or ${ }^{90}$ Y-labeled agents. The most dominant lesion (largest and with the most intense uptake) will overrule the other target lesions, and this representative lesion defines the overall SSTR-RADS score. With an overall SSTRRADS-1 to -3, PRRT with hot somatostatin analogs should not be considered, whereas for an overall SSTR-RADS-4 or -5, PRRT is recommended; however, common practical guidelines still apply $(22,43)$. In Supplemental Figures 1 and 2, SSTR-RADS has been applied to SSTR-PET/CT (Supplemental Fig. 1, overall SSTRRADS score of 5, and Supplemental Fig. 2, overall score of 3D).

\section{Potential Applications}

SSTR-RADS was formulated as a system to assess the baseline SSTR-targeted PET and gives recommendations for both further work-up and treatment. In this regard, it may guide the referring treating physician to consider PRRT with ${ }^{177} \mathrm{Lu}$ - or ${ }^{90}$ Y-labeled compounds. Moreover, it takes the level of uptake into account while using an internal organ reference. As part of MI-RADS, SSTR-RADS is based on the initial proposed framework system PSMA-RADS (although SSTR-targeted PET and NEN-specific details have been implemented in SSTR-RADS), and thus, both systems can be used reciprocally (23).

No validation studies with SSTR-RADS have yet been performed, unlike the NETPET grade, which has already proven its prognostic potential in a retrospective setting. When a dual-radiotracer approach of SSTR $/{ }^{18} \mathrm{~F}$-FDG PET/CT was combined in a single parameter, this framework system showed its capability as an outcome predictor (24). However, a dual-radiotracer approach is not performed routinely during follow-up, and thus, SSTR-RADS may serve as a reliable tool to investigate both baseline and follow-up scans. Its relevance for clinical practice is also evidenced by the recognition of pitfalls on SSTR-targeted PET/CT and by the associated recommendations for both work-up and treatment (e.g., indication for PRRT) (44).

Table 2 summarizes limitations and advantages of both systems for evaluating SSTR-targeted PET/CT.

\section{RECOMMENDATIONS FOR FUTURE VALIDATION AND A GLIMPSE AT FUTURE DIRECTIONS}

\section{Recommendations for Future Validation}

Before testing in real-world scenarios, the reproducibility and robustness of the standardized framework systems reviewed here should be proven (45). Thus, several steps for validation should be pursued. First, inter- and intraobserver agreement studies, ideally with multiple centers and multiple readers with different levels of experience, should be performed (27). Second, although it is not possible to biopsy every single investigated target lesion, indeterminate lesions should be identified and compared with follow-up imaging findings when pathologic results are not available (26). Third, validation with different radiotracers may be indispensable, because the biodistribution of PSMA radioligands, even with similar radioisotopes, may differ (e.g., the biodistribution among normal organs

TABLE 2

Head-to-Head Comparison of Standardized Framework Systems for SSTR-PET/CT for Evaluating NEN

\begin{tabular}{|c|c|c|}
\hline Standardized framework system & Advantages & Limitations \\
\hline NETPET grade (24) & $\begin{array}{l}\text { Has demonstrated its prognostic value in } \\
\text { retrospective study } \\
\text { Aims to combine dual-tracer approach in } \\
\text { single parameter } \\
\text { May also guide treatment, e.g., } \\
\text { somatostatin analogs for P1 or P2 vs. } \\
\text { chemotherapy for P5 } \\
\text { Discriminates subjects who have positive } \\
\text { uptake on both imaging modalities } \\
\text { (intermediate group, P2-P4) (42) } \\
\text { May be of value for risk stratification and } \\
\text { play potential role in clinical trials }\end{array}$ & $\begin{array}{l}\text { Does not routinely perform dual-tracer } \\
\text { approach during follow-up (44) } \\
\text { Is not baseline criterion } \\
\text { Does not consider pitfalls on SSTR-PET/CT } \\
\text { Gives fewer work-up recommendations for } \\
\text { specific situations, e.g., when to perform } \\
\text { biopsy or follow-up treatment }\end{array}$ \\
\hline SSTR-RADS, version 1.0 (22) & $\begin{array}{l}\text { Investigates baseline SSTR-PET/CT and } \\
\text { gives recommendations for both further } \\
\text { work-up and treatment } \\
\text { May guide referring treating physician to } \\
\text { consider PRRT } \\
\text { Is part of MI-RADS: SSTR-RADS and } \\
\text { PSMA-RADS can be applied reciprocally } \\
(19,23) \\
\text { Takes level of SSTR expression into } \\
\text { account (3-point qualitative assessment } \\
\text { to rate level of uptake) } \\
\text { Increases reader's confidence by } \\
\text { emphasizing pitfalls on SSTR-PET/CT } \\
\text { Is easy to memorize and use and, thus, may } \\
\text { be readily applicable in clinical setting }\end{array}$ & $\begin{array}{l}\text { Takes level of uptake into account while using } \\
\text { internal organ reference, but normal-organ } \\
\text { distribution may vary among novel SSTR } \\
\text { radiotracers (42) } \\
\text { Has arbitrary initial definitions and needs further } \\
\text { validation, e.g., interobserver agreement } \\
\text { studies and histopathologic comparisons }\end{array}$ \\
\hline
\end{tabular}


using either ${ }^{18}$ F-PSMA-1007 or ${ }^{18}$ F-DCFPyL) $(28,46)$. Fourth, validation of the detection rate of primary and metastatic disease with correlations to biopsy-driven histopathologic assessments are of importance to receive ultimate evidence (39). Fifth, the motivation to learn such standardized reporting systems should be evaluated; the change in a reader's confidence in interpreting PET/CT when such systems have been applied should be measured, with theranostic implications, and the implementation rate in clinical practice should be investigated. Finally, the frameworks reviewed here aim to provide structured reporting systems for both PCa and NEN; however, MI per se may still remain rather asemantic (i.e., the language used in a report needs to be conventionally associated to meanings) (47).

\section{Future Directions}

Much progress can be made toward more global standardization and rapid clinical implementation by creatively fusing the different existing systems-for example, by defining a single universal framework system per radiotracer under the umbrella of the different nuclear medicine societies. Such an approach may expedite transfer from leading institutions to smaller PET centers, open avenues for more tailored treatment decisions, allow for intra- and intercenter comparisons, and pave the way for adoption in multicenter studies.

Such framework systems should also be developed for other theranostic pairs, such as ${ }^{68} \mathrm{Ga}$-pentixafor $/{ }^{177} \mathrm{Lu}$-pentixather, which target the C-X-C chemokine receptor CXCR4 (6). Other potential theranostic twins may include the fibroblast activation protein-targeting ${ }^{68} \mathrm{Ga}-{ }^{90}$ Y-FAPI04 or the ${ }^{177} \mathrm{Lu}$-labeled and bombesin peptides for the gastrin-releasing peptide receptor $(5,7)$.

\section{DISCUSSION AND CONCLUSION}

Given the indispensable need for harmonization of the interpretation of PET radiotracers with potential theranostic applications, several framework systems for both PSMA-targeted PET for PCa and SSTR-targeted PET for NEN have been introduced in the last couple of years (19-24). Existing framework systems for interpretation of imaging findings with ${ }^{18} \mathrm{~F}-\mathrm{FDG}$ (Lugano, PERCIST) mainly allow for an overall assessment of treatment response, but theranostic radiotracers need baseline criteria to identify potential candidates for subsequent endoradiotherapies $(48,49)$. In addition, such criteria would be helpful to standardize collected data for large, multicenter trials (50). For PCa, 3 framework systems have been proposed: PROMISE provides an in-depth evaluation of imaging-based findings, in particular for anatomic details (e.g., primary tumor location on sextant basis) (20). In contrast, the 5-point scale of PSMA-RADS is easy to memorize and use and, thus, may serve as a powerful tool to assist in diagnosis in a busy clinical PCa practice (19). Moreover, PSMA-RADS has also been validated in a prospective interobserver agreement setting with overall high concordance rates, even among inexperienced readers (27). In a similar vein, the EANM consensus paper has also been further validated in a multicenter assessment, and consensus has been defined in a sophisticated approach conducting several Delphi rounds with expert readers (21).

For NEN, SSTR-RADS has recently been introduced, a system that is based on the PSMA-RADS system but takes SSTR-targeted PET- and NEN-specific details into account (22). Both PSMA- and SSTR-RADS have recently been summarized under a single umbrella term that describes the application of RADS for molecular oncology imaging (MI-RADS).
MI-RADS systems can be applied reciprocally; that is, imaging interpreters who are familiar with PSMA-RADS should be able to learn SSTR-RADS as well $(19,22,23)$. The NETPET system consolidates the findings of SSTR-targeted and ${ }^{18}$ F-FDG PET in a single parameter and provides treatment recommendations based on imaging findings (24).

Future studies are warranted to more completely validate such framework systems, such as interobserver agreement studies on a larger scale or correlations of imaging findings with histopathologic results $(27,39)$. In addition, consensus conferences are needed to further standardize these framework systems, ideally in a single universal framework system per radiotracer.

\section{DISCLOSURE}

Funding was provided by Progenics Pharmaceuticals, the Prostate Cancer Foundation Young Investigator Award, and National Institutes of Health grants CA134675, CA183031, CA184228, and EB024495. This project has also received funding from the European Union's Horizon 2020 Research and Innovation Program under Marie Sklodowska-Curie grant agreement 701983. Martin Pomper is a coinventor on a patent covering ${ }^{18} \mathrm{~F}-\mathrm{DCFPyL}$ and is entitled to a portion of any licensing fees and royalties generated by this technology. This arrangement has been reviewed and approved by the Johns Hopkins University in accordance with its conflict-of-interest policies. He has also received research funding from Progenics Pharmaceuticals, the licensee of ${ }^{18}$ F-DCFPyL. Michael Gorin has served as a consultant to Progenics Pharmaceuticals, and he, Kenneth Pienta, and Steven Rowe have received research funding from Progenics Pharmaceuticals. No other potential conflict of interest relevant to this article was reported.

\section{REFERENCES}

1. Young DS. Standardized reporting of laboratory data: the desirability of using SI units. N Engl J Med. 1974;290:368-373.

2. Leslie KO, Rosai J. Standardization of the surgical pathology report: formats, templates, and synoptic reports. Semin Diagn Pathol. 1994;11:253-257.

3. Barentsz JO, Weinreb JC, Verma S, et al. Synopsis of the PI-RADS v2 guidelines for multiparametric prostate magnetic resonance imaging and recommendations for use. Eur Urol. 2016;69:41-49.

4. Padhani AR, Lecouvet FE, Tunariu N, et al. METastasis reporting and data system for prostate cancer: practical guidelines for acquisition, interpretation, and reporting of whole-body magnetic resonance imaging-based evaluations of multiorgan involvement in advanced prostate cancer. Eur Urol. 2017;71:81-92.

5. Lindner T, Loktev A, Altmann A, et al. Development of quinoline based theranostic ligands for the targeting of fibroblast activation protein. $\mathrm{J} \mathrm{Nucl} \mathrm{Med}$. 2018;59:1415-1422.

6. Buck AK, Stolzenburg A, Hanscheid H, et al. Chemokine receptor-directed imaging and therapy. Methods. 2017;130:63-71.

7. Reynolds TS, Bandari RP, Jiang Z, Smith CJ. Lutetium-177 labeled bombesin peptides for radionuclide therapy. Curr Radiopharm. 2016;9:33-43.

8. Penet MF, Chen Z, Kakkad S, Pomper MG, Bhujwalla ZM. Theranostic imaging of cancer. Eur J Radiol. 2012;81(suppl 1):S124-S126.

9. Baum RP, Kluge AW, Kulkarni H, et al. $\left[{ }^{177} \mathrm{Lu}-\mathrm{DOTA}\right]^{0}$-D-Phe ${ }^{1}-\mathrm{Tyr}^{4}$-Octreotide $\left({ }^{177} \mathrm{Lu}\right.$-DOTATOC) for peptide receptor radiotherapy in patients with advanced neuroendocrine tumours: a phase-II study. Theranostics. 2016;6:501-510.

10. Kwekkeboom DJ, de Herder WW, Kam BL, et al. Treatment with the radiolabeled somatostatin analog $\left[{ }^{177} \mathrm{Lu}-\mathrm{DOTA}{ }^{0}, \mathrm{Tyr}^{3}\right]$ octreotate: toxicity, efficacy, and survival. J Clin Oncol. 2008;26:2124-2130.

11. Strosberg JR, Halfdanarson TR, Bellizzi AM, et al. The North American Neuroendocrine Tumor Society consensus guidelines for surveillance and medical management of midgut neuroendocrine tumors. Pancreas. 2017;46:707-714.

12. Strosberg J, El-Haddad G, Wolin E, et al. Phase 3 trial of ${ }^{177}$ Lu-dotatate for midgut neuroendocrine tumors. N Engl J Med. 2017;376:125-135. 
13. Rowe SP, Pomper MG, Gorin MA. Molecular imaging of prostate cancer: choosing the right agent. $J$ Nucl Med. 2018;59:787-788.

14. Hope TA, Afshar-Oromieh A, Eiber M, et al. Imaging prostate cancer with prostate-specific membrane antigen PET/CT and PET/MRI: current and future applications. AJR. 2018;211:286-294.

15. Afshar-Oromieh A, Holland-Letz T, Giesel FL, et al. Diagnostic performance of ${ }^{68}$ Ga-PSMA-11 (HBED-CC) PET/CT in patients with recurrent prostate cancer: evaluation in 1007 patients. Eur J Nucl Med Mol Imaging. 2017;44:1258-1268.

16. Derlin T, Schmuck S, Juhl C, et al. Imaging characteristics and first experience of $\left[{ }^{68} \mathrm{Ga}\right]$ THP-PSMA, a novel probe for rapid kit-based Ga-68 labeling and PET imaging: comparative analysis with $\left[{ }^{68} \mathrm{Ga}\right]$ PSMA I\&T. Mol Imaging Biol. 2018;20: $650-658$.

17. Meignan M, Gallamini A, Meignan M, Gallamini A, Haioun C. Report on the First International Workshop on Interim-PET-Scan in Lymphoma. Leuk Lymphoma. 2009;50:1257-1260.

18. Wahl RL, Jacene H, Kasamon Y, Lodge MA. From RECIST to PERCIST: evolving considerations for PET response criteria in solid tumors. J Nucl Med. 2009;50(suppl 1):122S-150S.

19. Rowe SP, Pienta KJ, Pomper MG, Gorin MA. Proposal for a structured reporting system for prostate-specific membrane antigen-targeted PET imaging: PSMARADS version 1.0. J Nucl Med. 2018;59:479-485.

20. Eiber M, Herrmann K, Calais J, et al. Prostate Cancer Molecular Imaging Standardized Evaluation (PROMISE): proposed miTNM classification for the interpretation of PSMA-ligand PET/CT. J Nucl Med. 2018;59:469-478.

21. Fanti S, Minozzi S, Morigi JJ, et al. Development of standardized image interpretation for ${ }^{68} \mathrm{Ga}$-PSMA PET/CT to detect prostate cancer recurrent lesions. Eur J Nucl Med Mol Imaging. 2017;44:1622-1635.

22. Werner RA, Solnes LB, Javadi MS, et al. SSTR-RADS version 1.0 as a reporting system for SSTR PET imaging and selection of potential PRRT candidates: a proposed standardization framework. J Nucl Med. 2018;59:1085-1091.

23. Werner RA, Bundschuh RA, Bundschuh L, et al. MI-RADS: molecular imaging reporting and data systems: introduction of PSMA- and SSTR-RADS for radiotracers with potential theranostic implications. Ann Nucl Med. 2018;32: 512-522.

24. Chan DL, Pavlakis N, Schembri GP, et al. Dual somatostatin receptor/FDG PET/ CT imaging in metastatic neuroendocrine tumours: proposal for a novel grading scheme with prognostic significance. Theranostics. 2017;7:1149-1158.

25. Sheikhbahaei S, Afshar-Oromieh A, Eiber M, et al. Pearls and pitfalls in clinical interpretation of prostate-specific membrane antigen (PSMA)-targeted PET imaging. Eur J Nucl Med Mol Imaging. 2017;44:2117-2136.

26. Yin Y, Werner RA, Higuchi T, et al. Follow-up of lesions with equivocal radiotracer uptake on PSMA-targeted PET in patients with prostate cancer: predictive values of the PSMA-RADS-3A and PSMA-RADS-3B categories. J Nucl Med. September 6, 2018 [Epub ahead of print].

27. Werner RA, Bundschuh RA, Bundschuh L, et al. Interobserver agreement for the standardized reporting system PSMA-RADS 1.0 on ${ }^{18} \mathrm{~F}$-DCFPyL PET/CT imaging. J Nucl Med. 2018;59:1857-1864.

28. Giesel FL, Hadaschik B, Cardinale J, et al. F-18 labelled PSMA-1007: biodistribution, radiation dosimetry and histopathological validation of tumor lesions in prostate cancer patients. Eur J Nucl Med Mol Imaging. 2017;44:678-688.

29. Werner RA, Sheikhbahaei S, Jones KM, et al. Patterns of uptake of prostatespecific membrane antigen (PSMA)-targeted ${ }^{18} \mathrm{~F}$-DCFPyL in peripheral ganglia. Ann Nucl Med. 2017;31:696-702.
30. Rischpler C, Beck TI, Okamoto $\mathrm{S}$, et al. ${ }^{68} \mathrm{Ga}$-PSMA-HBED-CC uptake in cervical, celiac and sacral ganglia as an important pitfall in prostate cancer PET imaging. J Nucl Med. 2018;59:1406-1411.

31. Fendler WP, Eiber M, Beheshti M, et al. ${ }^{68}$ Ga-PSMA PET/CT: joint EANM and SNMMI procedure guideline for prostate cancer imaging: version 1.0. Eur J Nucl Med Mol Imaging. 2017;44:1014-1024.

32. De Coster L, Sciot R, Everaerts W, et al. Fibrous dysplasia mimicking bone metastasis on ${ }^{68}$ GA-PSMA PET/MRI. Eur J Nucl Med Mol Imaging. 2017;44: 1607-1608.

33. Gykiere P, Goethals L, Everaert H. Healing sacral fracture masquerading as metastatic bone disease on a ${ }^{68} \mathrm{Ga}-\mathrm{PSMA}$ PET/CT. Clin Nucl Med. 2016;41: e346-e347.

34. Ardies PJ, Gykiere P, Goethals L, De Mey J, De Geeter F, Everaert H. PSMA uptake in mediastinal sarcoidosis. Clin Nucl Med. 2017;42:303-305.

35. Vamadevan S, Shetty D, Le K, Bui C, Mansberg R, Loh H. Prostate-specific membrane antigen (PSMA) avid pancreatic neuroendocrine tumor. Clin Nucl Med. 2016;41:804-806.

36. Lawhn-Heath C, Flavell RR, Glastonbury C, Hope TA, Behr SC. Incidental detection of head and neck squamous cell carcinoma on ${ }^{68} \mathrm{Ga}-\mathrm{PSMA}-11 \mathrm{PET} / \mathrm{CT}$. Clin Nucl Med. 2017;42:e218-e220.

37. Rowe SP, Gorin MA, Hammers HJ, et al. Imaging of metastatic clear cell renal cell carcinoma with PSMA-targeted ${ }^{18}$ F-DCFPyL PET/CT. Ann Nucl Med. 2015;29: 877-882.

38. Gorin MA, Rowe SP, Hooper JE, et al. PSMA-targeted ${ }^{18}$ F-DCFPyL PET/CT imaging of clear cell renal cell carcinoma: results from a rapid autopsy. Eur Urol. 2017;71:145-146

39. Cho SY. Proposed criteria positions PSMA PET for the future. J Nucl Med. 2018;59:466-468.

40. Lückerath K, Stuparu AD, Wei L, et al. Detection threshold and reproducibility of ${ }^{68} \mathrm{Ga}$-PSMA11 PET/CT in a mouse model of prostate cancer. J Nucl Med. 2018;59:1392-1397.

41. Bennett C, Vakil N, Bergman J, et al. Consensus statements for management of Barrett's dysplasia and early-stage esophageal adenocarcinoma, based on a Delphi process. Gastroenterology. 2012;143:336-346.

42. Hindié E. The NETPET score: combining FDG and somatostatin receptor imaging for optimal management of patients with metastatic well-differentiated neuroendocrine tumors. Theranostics. 2017;7:1159-1163.

43. Bodei L, Mueller-Brand J, Baum RP, et al. The joint IAEA, EANM, and SNMMI practical guidance on peptide receptor radionuclide therapy (PRRNT) in neuroendocrine tumours. Eur J Nucl Med Mol Imaging. 2013;40:800-816.

44. Arnold R, Chen YJ, Costa F, et al. ENETS consensus guidelines for the standards of care in neuroendocrine tumors: follow-up and documentation. Neuroendocrinology. 2009;90:227-233.

45. Gennaro G. The "perfect" reader study. Eur J Radiol. 2018;103:139-146.

46. Szabo Z, Mena E, Rowe SP, et al. Initial evaluation of $\left[{ }^{18} \mathrm{~F}\right] \mathrm{DCFPyL}$ for prostatespecific membrane antigen (PSMA)-targeted PET imaging of prostate cancer. Mol Imaging Biol. 2015;17:565-574.

47. Willis BH, Beebee H, Lasserson DS. Philosophy of science and the diagnostic process. Fam Pract. 2013;30:501-505.

48. Johnson SA, Kumar A, Matasar MJ, Schoder H, Rademaker J. Imaging for staging and response assessment in lymphoma. Radiology. 2015;276:323-338.

49. JH O, Lodge MA, Wahl RL. Practical PERCIST: a simplified guide to PET response criteria in solid tumors 1.0. Radiology. 2016;280:576-584.

50. Ceci F, Fanti S. Standardisation of PSMA images interpretation: why do we need it? Clin Transl Imaging. 2018; 6:331-333. 